\title{
Vigilância Epidemiológica das Intoxicações Exógenas Atendidas em um Hospital de Ensino
}

RESUMO | Objetivo: Descrever o perfil das intoxicações exógenas atendidas em um hospital de ensino, no período de junho de 2015 a junho de 2019. Método: Estudo documental, retrospectivo, descritivo, com análise quantitativa. A coleta dos dados ocorreu no Sistema de Informação de Agravo de Notificação (SINAN), do período de junho de 2015 a junho de 2019. O total da amostra foi 152 notificações exógenas. Resultados: Quanto ao sexo predominou o feminino com 85 casos, houve predomínio para as idades, entre 20 a 34 anos, para o local de exposição, 132 casos aconteceram na residência, nos anos de 2017 e 2018 apresentaram maior incidência. Foram identificados 127 agentes toxicológicos dentre esses 98 casos, os anticoagulantes, benzodiazepínicos e antidepressivos tiveram maior incidência. Conclusão: Jovem adulto nas idades entre 20 a 34 anos, principalmente do sexo feminino predominam nas notificações de intoxicação exógena, com maior concentração para as pessoas com nível escolar incompleto.

Palavras-chaves: Intoxicação, Vigilância epidemiológica, Notificação.

\begin{abstract}
Objective: Describe the profile of exogenous intoxication attended in a teaching hospital, between June 2015 and June 2019. Method: Documental, retrospective, descriptive study, with qualitative analysis. The data collection was done on the Information System of Notification Aggravation (SINAN), between June 2015 and June 2019. The total sample was 152 exogenous notifications. Results: The predominant gender was the female, with $85 \mathrm{cases}$, there was the predominance of the ages between 20 and 34 years old, in the local of exposition, 132 cases took place in the recidence, in 2017 and 2018 there was a higher incidence. 127 toxicological agents were identified among these 98 cases, the anticoagulants, benzodiazepines, and antidepressants had higher incidence. Conclusion: Young adults aged between 20 and 34 years old, especially of the female gender are predominant in notifications of exogenous intoxication, with bigger concentration of people with incomplete educational attainment.
\end{abstract}

Keywords: Intoxication, Epidemiological surveillance, Notification.

RESUMEN | Objetivo: Describir el perfil de intoxicaciones exógenas atendidas en un hospital docente, en el período de junio de 2015 a junio de 2019. Método: Estudio documental, retrospectivo, descriptivo, con análisis cuantitativo. La recopilación de datos tuvo lugar en el Sistema de Información de Divulgación Notificable (SINAN), en el periodo de junio de 2015 a junio de 2019. El total de la muestra fueron 152 notificaciones exógenas. Resultados: En cuanto al género, hubo un predominio de mujeres con 85 casos, hubo un predominio de edades, entre 20 y 34 años, para el lugar de exposición, 132 casos ocurrieron en la residencia, en los años 2017 y 2018 tuvieron una mayor incidencia. Se identificaron 127 agentes toxicológicos entre estos 98 casos, los anticoagulantes, las benzodiacepinas y los antidepresivos tuvieron una mayor incidencia. Conclusión: los adultos jóvenes entre 20 y 34 años, principalmente mujeres, predominan en las notificaciones de intoxicación exógena, con mayor concentración para las personas con escolarización incompleta.

Palabras claves: Intoxicación; Vigilancia epidemiológico; Notificación.

\section{Maristela Salete Maraschin}

Enfermeira. Docente da Universidade Estadual do Oeste do Paraná/UNIOESTE, campus Cascavel/PR.

ORCID: 0000-0003-2184-5056

\section{Sabrina de Kássia Meneguesso Carmello \\ Enfermeira. Residente do Programa de Re- sidência em Vigilância em Saúde e Con- trole de Infecção. Hospital Universitário do Oeste do Paraná/HUOP/Universidade Estadual do Oeste do Paraná/UNIOESTE, campus Cascavel/PR. \\ ORCID: 0000-0003-4343-4805}

\section{Leda Aparecida V. Nabuco de Gouvêa}

Enfermeira. Docente da Universidade Estadual do Oeste do Paraná/UNIOESTE, campus de Cascavel/PR.

ORCID: 0000-0001-6641-7114

\section{Cláudia Ross}

Enfermeira. Docente da Universidade Estadual do Oeste do Paraná/UNIOESTE, campus de Cascavel/PR.

ORCID: 000-0001-6641-7114

\section{Fabiana Severino Kupka}

Enfermeira. Vigilância Epidemiológica Hospitalar. Hospital Universitário do Oeste do Paraná/HUOP. Cascavel/PR.

ORCID: 0000-0002-3687-1241

Recebido em: 27/02/2020

Aprovado em: 06/07/2020
INTRODUÇÃO

$\Lambda$ intoxicação exógena é caracterizada por sintomas clínicos causados pela ingestão de substância tóxica, podendo ser acidental, superdosagem, interação química ou algumas vezes por tentativa de suicídio(1). A etiologia da intoxicação pode advir de várias fontes, como, ingestão de alimentos tóxicos ou contaminados, medicamentos, uso de agrotóxicos, causada por fatores patológicos ou fatores exógenos causados pelo ambiente por ${ }^{(2,3)}$.

As atenções no atendimento as intoxicações exógenas desempenham papel importante para o desencadeamento de ações voltadas para sua prevenção devido à grande influência direta para mor- 
bimortalidade do agravo. Estima-se que 1,5 a $3 \%$ da população sofre algum tipo de exposição a agentes tóxicos, e que o Brasil apresenta 4.800.000 casos anuais de intoxicações exógenas ${ }^{(4,5,6)}$.

No Brasil estima-se que $60 \%$ das intoxicações exógenas são causadas por ingestão de medicamentos, sendo que, a ingestão proposital por tentativa de suicídio sobressai-se e as acidentais ocorrem com mais frequência em crianças menores de três anos de idade, caracterizadas por sua vulnerabilidade ${ }^{(7)}$.

A Portaria número 2.472, de 31 de agosto de 2010, define por intoxicação aquela causada por substâncias químicas, incluindo agrotóxicos, gases tóxicos e metais pesados, sendo agravos e eventos em saúde pública de notificação compulsória em todo o território nacional e registrado no Sistema de Informação de Agravos de Notificação (SINAN) ${ }^{(7)}$.

Acerca das intoxicações, existem os centros de assistência toxicológica que prestam atendimentos diários a fim de orientar ações e treinar profissionais da área da saúde objetivando sistematizar condutas e servir como contrarreferência à serviços da saúde divulgando pesquisas, experiências e protocolos, para nortear as ações nas medidas promocionais e de reabilitação do indivíduo intoxicado. Implantados desde 1986, os Centros de Informação Toxicológica (Ceatox) ${ }^{(8,9)}$.

O conhecimento epidemiológico o qual aborda variáveis de identificação dos casos notificados é de grande importância para a saúde, pois norteia as ações de prevenção, promoção e reabilitação. Ante o exposto, questionou-se: Qual é o perfil das intoxicações exógenas atendidas em um hospital de ensino? Para tanto, este estudo teve como objetivo descrever o perfil das intoxicações exógenas atendidas nesse hospital, no período de junho de 2015 a junho de 2019.

\section{MÉTODO}

Estudo descritivo, retrospectivo e documental, com análise quantitativa de ca- sos notificados por intoxicação exógena atendidos em um hospital escola e notificados pelo Núcleo de Vigilância Epidemiológica Hospitalar (NVEH).

Os dados foram coletados junto no Sistema de Informação de Agravos de Notificação (SINAN) de todas as fichas de notificação de intoxicação exógena no-

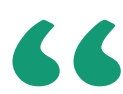

No Brasil estimase que $60 \%$ das intoxicações exógenas são causadas por ingestão de medicamentos, sendo que, a ingestão proposital por tentativa de suicídio sobressaise e as acidentais ocorrem com mais frequência em crianças menores de três anos de idade

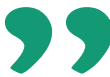

tificadas no período de junho de 2015 a junho de 2019. Para a coleta de dados foi elaborado um roteiro, dividido em duas partes, a primeira parte foi sobre as características sociodemográficas, tais como: sexo, idade, raça/cor, escolaridade, e ocupação/profissão, a segunda foi relativa as características das intoxicações exógenas, como: local de exposição, agente toxico- lógico, princípio ativo do agente toxicológico, veículo de contaminação e evolução do caso. Todas as variáveis selecionadas estão compostas na ficha de notificação obrigatória de intoxicação exógena. A coleta de dados foi realizada no mês juIho de 2019. No período do estudo foram notificados 152 casos, sendo este o total da amostra. Não houve exclusão de casos notificados, pois as variáveis selecionadas para este estudo estavam completamente preenchidas no SINAN.

Este estudo faz parte do projeto, denominado "Caracterização do Perfil das Doenças e Agravos de Notificações Obrigatória e das Infecções Relacionadas à Assistência em Saúde (IRAS)" o qual foi aprovado pelo Comitê de Ética e Pesquisa que envolve Seres Humanos, da Universidade Estadual do Oeste do Paraná/UNIOESTE, conforme parecer número 2.751.985 e CAAE 90600318.3.0000.0107, respeitando os princípios da Resolução 466/2012 da Comissão Nacional de Ética e Pesquisa Envolvendo Seres Humanos ${ }^{(10)}$.

Os dados obtidos foram sumarizados em planilhas eletrônicas do software Microsoft Office Excel@ versão 2007 e, posteriormente, analisados por estatística descritiva, obtida pelas medidas de frequência relativa e absolutas dos casos notificados e posteriormente analisados e dispostos em tabela.

\section{RESULTADOS}

No período do estudo, foram notificados 152 casos de intoxicação exógena, predominando o sexo o feminino com 85 $(55,92 \%)$ dos casos e masculino com 67 $(44,07 \%)$.

Alusivo à idade, menor de um ano cinco casos $(3,28 \%)$, de um a quatro anos $17(11,18 \%)$, cinco a nove anos sete casos (4,60\%), 10 a 14 anos com 16 (10,52\%), 15 a 19 anos 15 (9,86\%), 20 a 24 com $18(11,84 \%), 25$ a 29 casos $12(7,89 \%)$, 30 a 34 casos sete $(4,60 \%), 35$ a 39 com nove $(5,92 \%), 40$ a 44 com nove $(5,92 \%)$, 45 a 49 com nove (5,26\%), 50 a 54 com sete $(4,60 \%), 55$ a 59 anos dois $(1,31 \%)$, 
60 a 64 anos três (1,97\%), 65 a 69 com dois (1,31\%), 70 a 74 anos um (0,65\%), 75 a 79 com oito (5,26\%) e 80 anos e mais com 10 casos 10 (6,57\%). Quanto a raça/cor, $124(81,57 \%)$ se declararam da cor branca, 20 (13,15\%) da cor parda, seis $(3,94 \%)$ da cor preta, da cor amarela e indígena um $(0,65 \%)$ cada um.

Em relação a escolaridade, o ensino fundamental incompleto teve 51 casos $(33,55 \%)$, fundamental completo 14 casos $(9,21 \%)$, ensino médio incompleto 20 casos $(13,15 \%)$, ensino médio completo 22 casos $(14,47 \%)$, curso superior incompleto 4 casos $(2,63 \%)$, curso superior completo $5(3,28 \%)$, analfabetos 3 (1,97\%), ignorado/branco e/ou não se aplica 33 casos $(21,71 \%)$.

Concernente as ocupações/profissões dos indivíduos notificados, 32 casos eram $(21,05 \%)$ estudantes, 26 casos $(17,10 \%)$ donas de casa, 21 casos (13,81\%) aposentados, 12 casos $(7,89 \%)$ agricultores, 10 $(18,18 \%)$ ignorados. Os demais percentuais distribuíram-se a diferentes profissões, totalizando 34 casos $(22,36 \%)$, destacando-se: auxiliar administrativo; atendente de farmácia; estagiário; vendedor/logista; auxiliar de produção; professor; operacasos $(6,57 \%)$ desempregados e 17 casos dor de máquina; marceneiro; pedreiro; balconista; autônomo; atendente de pet-shop e cabelereiro.

Relativo ao local de exposição, 132 $(86,84 \%)$ das intoxicações ocorreram na residência. Sete casos $(4,60 \%)$ no ambiente de trabalho, seis (3,94\%) em ambiente externo, quatro $(2,63 \%)$ em outros locais, dois (1,31\%) em serviços de saúde e um $(0,65 \%)$ ignorado/branco.

A tabela 1 apresenta os dados sobre os agentes toxicológicos, conforme ano de distribuição.

Os anos de 2017 e 2018 apresentarem o maior número de intoxicações sendo respectivamente 43 (28,28\%) 45 $(29,60 \%)$. Em relação ao agente toxicológico, predominaram as intoxicações por medicamentos 98 (64,47\%), não possuindo variância significativa entre os demais agentes toxicológicos.

Quanto aos princípios ativos dos agentes, foram contabilizados 127 princípios ativos, que foram organizados em classes terapêuticas conforme a ficha de notificação, com predomínio dos anticoagulantes que teve $42(27,63 \%)$ casos, seguida dos benzodiazepínicos com 24 (15,78\%), antidepressivo, 18 (11,84\%), os anticonvulsivantes $16(10,52 \%)$ e os anti-inflamatórios 12 (7,89\%) casos.

O tipo de exposição predominante foi a aguda/única totalizando 117 (76,97\%) seguida de aguda/repetida com $24(15,78 \% /)$ casos. O tipo crônico teve seis $(3,94 \%)$ casos, enquanto aguda sub crônica totalizou três (1,97\%), ignorado/ branco dois (1,31\%). Relativo ao veículo de contaminação, a tentativa de suicídio prevaleceu com 71 (46,71\%) casos, o uso acidental apresentou 36 (23,68\%) casos seguido de intoxicação por uso terapêutico 17 (11,18\%). Erro de administração e abuso ambos apresentaram sete (4,60\%) casos cada um, o uso habitual configurou seis $(3,94 \%)$, a automedicação teve três $(1,97 \%)$ casos, prescrição médica dois $(1,31 \%)$, violência/homicídio, tentativa de aborto e ingestão de alimento apresentaram um caso para cada $(0,65 \%)$.

Quanto a evolução do caso, este estudo mostrou que a cura sem sequela predominou com 128 (84,21\%) casos, 12 (7,89\%) evoluíram para óbito, sendo óbito pela intoxicação ou óbito por outras causas, três casos $(1,97 \%)$ constavam como ignorado/branco, cinco $(3,28 \%)$ tiveram perda de segmento e quatro $(2,63 \%)$ tiveram cura com sequela.

\section{DISCUSSÃO}

TABELA 1 - Ocorrência de intoxicação exógena quanto ao tipo de agente toxicológico da exposição, no período de junho de 2015 a junho de 2019. Cascavel/PR. 2019.

\begin{tabular}{lcccccc}
\hline AGENTE TÓXICO & 2015 & 2016 & 2017 & 2018 & 2019 & TOTAL \\
\hline Medicamento & 8 & 16 & 26 & 34 & 14 & 98 \\
\hline Agrotóxico agrícola & 3 & 1 & 3 & 2 & 2 & 11 \\
\hline Agrotóxico doméstico & 1 & 3 & 0 & 0 & 0 & 4 \\
\hline Raticida & 1 & 0 & 2 & 2 & 0 & 5 \\
\hline Produto veterinário & 0 & 1 & 1 & 0 & 0 & 2 \\
\hline Produto uso domiciliar & 2 & 0 & 1 & 1 & 1 & 5 \\
\hline Cosmético & 1 & 0 & 0 & 0 & 0 & 1 \\
\hline Produto químico & 2 & 2 & 4 & 5 & 1 & 14 \\
\hline Droga de abuso & 0 & 0 & 4 & 1 & 3 & 8 \\
\hline Alimento e bebida & 0 & 0 & 0 & 0 & 1 & 1 \\
\hline IGN/BRA & 0 & 0 & 2 & 0 & 1 & 3 \\
\hline TOTAL & 18 & 23 & 43 & 45 & 23 & 152
\end{tabular}

Fonte: SINAN/2019.
Quando relacionados à idade o estudo teve discordância entre as faixa-etárias quando comparado a outros estudos, os quais destacaram as idades entre 20 a 40 anos um percentual de 30,3 a 54,1\% para essa faixa etária, nos quais pessoas com idade superior a 40 totalizaram 40,5\% das notificações e em outro estudo as idades que prevaleciam entre 16 a 25 anos com percentual de 32\% (1,2-5,5-8). Pode-se observar que no presente estudo essa faixa etária prevaleceu, mas também se observa um grande número para idades entre um a quatro anos 17 (11,18\%).

O estudo evidenciou que a tentativa de suicídio teve prevalência entre indivíduos de 20 a 34 idade. Dados semeIhantes foram encontrados em um estudo multicêntrico realizado na Suíça, que 
relatou que as tentativas de suicídio por envenenamento foram mais comuns entre os adolescentes e adultos jovens ${ }^{(9)}$.

Outro estudo que analisou os casos de intoxicação aguda por agrotóxicos no Brasil, em um período de cinco anos, mostrou as idades entre 20 a 39 anos apresentaram 20.630 intoxicações, com percentual de $46 \%$ das notificações ${ }^{(11)}$.

Quanto à raça/cor, poucos estudos trazem essa variável em suas análises. Um estudo apresentou essa variável, no qual $6.821(66,4 \%)$ dos seus casos eram em pessoas declaradas pardas (8). Estudos retrospectivos com uso de prontuários, fichas ou formulários de atendimento previamente preenchidos por funcionários diferentes, principalmente por terceiros ao atendimento, apresentam viés relacionado ao preenchimento, perdas de informações pertinentes e algumas vezes o preenchimento dos campos constava como ignorado.

Frente a presença de campos com a variável ignorados questiona-se a possibilidade de negligência no preenchimento dos dados ou de fornecimento incompleto de informações por parte dos pacientes ${ }^{(5)}$.

A predominância da residência como local de exposição aparece na maioria dos estudos como o principal local de exposição variando entre 56,8 a 85,7\%, dados que corroboram com o estudo em questão. Na maioria dos casos apresentavam-se em localidade geográfica predominantemente a urbana, afirmado em um dos estudos por um percentual de 1.046 $(64 \%)$ dos $\operatorname{casos}^{(2-5-11,12) \text {. }}$

Este estudo mostra que o dado epidemiológico sobre o agente toxicológico causal anticoagulante é um dado isolado, evidenciado em outros estudos que os Benzodiazepínicos predominam. Um estudo realizado no período de 1998 a 2011 analisou as intoxicações por medicamentos, sendo um total de 16.774 acidentes por ingestão deste agente, teve o percentual de 32,5\% dos atendimentos por intoxicação exógena no período (13). Este estudo caracterizou a classe medicamentosa, sendo que os Benzodia-
Outro estudo que analisou os casos de intoxicação aguda por agrotóxicos no Brasil, em um período de cinco anos, mostrou as idades entre 20 a 39 anos apresentaram 20.630 intoxicações, com percentual de $46 \%$ das notificações. zepínicos foi o maior agente causador da intoxicação exógena com percentual de $(17,2 \%)$ das intoxicações ${ }^{(14)}$.

Estudo realizado entre os anos de 2006 a 2011 em um hospital do Irã registrou 108.265 casos de intoxicação por medicamentos. Quando analisados os dados referente ao intoxicante os antiepilépticos, sedativo-hipnótico e drogas antiparkinsoniano tiveram prevalência em com 24,185 (22.3\%) das medicações identificadas $^{(15)}$.

$\mathrm{Na}$ maioria dos casos a ingestão de medicamentos está relacionada ao pensamento suicida. No acompanhamento a 153 casos de intoxicação exógena entre 2009 a 2011 em um hospital de alta complexidade na Turquia, mostrou que $135(71,2 \%)$ foram por ingestão de medicamentos e 144 (94,1\%) eram por tentativa suicida (16). Os dados se equiparam ao quando comparados ao presente estudo, que evidenciou a tentativa de suicídio com 71 (46,71\%) dos casos. No Brasil os dados de suicídio aumentaram, entre os anos de 2007 a 2016. Quando foi registrado 106.374 mortes em decorrência do suicídio, pela intoxicação por medicamentos responsável por $18 \%$ das mortes ${ }^{(17)}$.

Com relação ao agente causal, 13.322 (7.3\%) foram por medicação, os Benzodiazepínicos 4344 (83.2\%) teve prevalência entre as classes farmacológicas, entre eles o Diazepam 2.374 (45.5\%). Neste estudo o uso terapêutico configurou $44 \%$ das intoxicações. O uso acidental apresentou 36 (23,68\%) seguido de intoxicação por uso terapêutico 17 $(11,18 \%)$.

Em um estudo de cinco anos, caracterizou um total de 277 vítimas de intoxicação exógena, 215 (77,6\%) tiveram a intoxicação aguda como principal tipo de exposição, seguida de 34 (12,3\%) aguda repetida (18). Estes dados corroboram com os encontrados neste estudo, sendo que a aguda única 117 (76,97\%) casos e a repetida $24(15,78 \%)$.

Dados de outro estudo frente ao desfecho dos casos, em dois anos mostraram que $86(72,8 \%)$ tiveram cura sem sequela 
e $14(11,8 \%)$ evoluíram para óbito pela intoxicação ${ }^{(19)}$. O presente estudo encontrou $84,21 \%$ do total da amostra pesquisada, evoluiu para cura sem sequelas e $7,89 \%$ evoluíram para óbito. A mortalidade de pessoas intoxicadas foi baixa, esses dados são apresentados também em um estudo que analisou o desfecho dos casos de intoxicação, sendo que variam entre 0 a 1,4\% dos pacientes admitidos com o diagnóstico de intoxicação exógena ${ }^{(20)}$.

\section{CONCLUSÃO}

O estudo teve como achado relevante que jovens adultos entre as idades 20 a 34 anos, principalmente do sexo feminino preponderou nas notificações de intoxicação exógena, concentrado nas pessoas com nível escolar incompleto. Determinantes sociais os quais expõem as vítimas a desenvolver problemas psicológicos, associados aos determinantes físicos que são influenciados na grande maioria das vezes pela terminalidade das condições da qualidade de vida e dos fatores econômicos, possibilitam as vítimas cometer suicídio.

A subnotificação ainda é um problema que permeia os estudos de característica epidemiológica, pois a ausência de informações pode contribuir para resul- tados não representativos. A notificação dos casos de agravos irá construir indicadores que servirão de base para o planejamento de ações, as quais irão contribuir para implementação de medidas para a melhorias dos indicadores de saúde.

Sugere-se o desenvolvimento de novos estudos epidemiológicos acerca das intoxicações exógenas em outras realidades, a fim de consolidar informações evidenciadas na prática, que possam fortalecer políticas públicas frente a este agravo.

Espera-se que novos estudos possam ser desenvolvidos em outros cenários, para que os resultados sejam agregados aos encontrados neste estudo.

\section{Referências}

1. Almeida, TCA; Couto, CC; Cequer, FMD. Perfil das intoxicações agudas ocorridas em uma cidade do centro-oeste de minas gerais. Eletronic JournalOfPharmacy 2016; 8 (3):151-162. DOI: https://doi.org/10.5216/ref. v13i3.39923.

2. Santos, RR; Almeida, OPN; Cunha, CM. Perfil de vítimas de intoxicações exógenas agudas e assistência de enfermagem. Rev. de Enferma. e Atenção à Saúde 2015; 4(2):45-55. ISSN 2317-1154.

3. Oliveira, FFS; Suchara, EA. Perfil epidemiológico das intoxicações exógenas em crianças e adolescentes em município do Mato Grosso. Rev. Paulista de Pediatria 2014; 32(4): 299-305. DOI: http://dx.doi.org/10.1016/j. rpped.2014.06.002.

4. Baril, MS; Chakraborty SR; Alam, MMJ; Qayyum, JÁ; Hassan, N; Chowdhury, FR. Four-YearStudyonAcutePoisoning Cases Admittedto a Tertiary Hospital in Bangladesh: Emerging Trend ofPoisoning in Commuters.Emerging Trend ofPoisoning in Commuters in Bangladesh 2014; 3(4): 152:156. DOI: 10.22038/APJMT.2014.3485.

5. Toscano, MM; Ladim, JT; Rocha, AB; Munõz, RLS. Intoxicações exógenas agudas registradas em centro de assistência toxicológica. Rev. Saúde e Pesquisa 2016; 9(3) :425-432. ISSN:1983-1870.

6. Oliveira, DH; Suchara, EA. Intoxicações medicamentosas em hospital público de Barra do Garças -MT, no período de 2006 a 2009. Rev. Ciênc. Méd. Biol.; 2014: 13(1): 55-59. ISSN 1677-5090.

7. Ministério da Saúde (BR). Portaria Número 204, de 17 de fevereiro de 2016. Lista Nacional de Notificação Compulsória de doenças, agravos e eventos de saúde pública nos serviços de saúde públicos e privados em todo o território nacional. Brasília: MS; 2016.

8. Liberato, AA; Freire, LS; Lobo, PHP; Dias, FCF; Guedes, VR. Intoxicações exógenas na região norte: atualização clínica e epidemiológica. Revista de Patologia do Tocantins 2017; 4(2): 61-64. DOI: 10.20873/uft.2446-6492.2017v$4 \mathrm{n} 2 \mathrm{p} 61$.

9. Pires, MCC; Silva, TPS; Passos, MP; Sougey, EB; Filho, OCB. Risk factorsof suicide attemptsbypoisoning: review. TrendsPsychiatryPsychother [Internet] 2014; 36(2): 63-74[acesso em 17 jun 2019]. DOI: http://dx.doi. org/10.1590/2237-6089-2013-0044.

10. Ministério da Saúde (BR). Conselho Nacional de Saúde. Resolução 466/2012. Dispõe sobre normas e de pesquisa com seres humanos. Brasília: DF; 2012.

11. Matos, AS. Análise das intoxicações exógenas por agrotóxicos no Brasil, entre 2007 a 2012. 2013. 50 f. Dissertação (Mestrado) - Curso de Enfermagem, Universidade de Brasília, 2013.

12. Vieira, LP; Santana, VTP, Suchara, EA. Caracterização de tentativas de suicídios por substâncias exógenas. Cad. Saúde Colet 2015; 23(2): 118-123. DOI: 10.1590/1414-462X201500010074.

13. Vallersnes, OM; Jacobsen, D; Ekeberg, Ø; Brekke, M. Patientspresentingwithacutepoisoningtoanoutpatientemergencyclinic: a one-yearobservationalstudy in Oslo, NorwayVallersnes. BMC Emergency Medicine 2015; 1(1): 15-18. DOI 10.1186/s12873-015-0045-2

14. Costa, AOC; Alonzo, HGA. Casos de exposições e Intoxicações por medicamentos registrados em um Centro de Controle de intoxicações do interior do Estado de São Paulo. Rev. Bras. Pesq. Saúde, Vitória 2015; 17(2): 52-60. Disponível em: http://periodicos.ufes.br/RBPS/article/download/13188/9238. 15. Hassanian-Moghaddam, H; Zamani, N; Rahimi, M; Shadnia, S; Pajoumand, A; Sarjami, S. AcuteAdultandAdolescentPoisoning in Tehran, Iran; theEpidemiologic Trend between 2006 and 2011. Archivesoflranian Medicine 2014; 17(8): 534-538. Disponível em: http://www.ams.ac.ir/AIM/ NEWPUB/14/17/8/003.pdf.

16. Yaylacl, S; Genç, AB; Demir, MV; Cinemre, H; Tamer, A. Retrospectiveevaluationofpatientsat follow-up withacutepoisoning in IntensiveCare Unit. NigerianJournalofClinical Practice2016; 223-226. DOI: 10.4103/11193077.164340.

17. Brasil. Ministério da Saúde. Boletim Epidemiológico. Suicídio, Saber, Agir e Prevenir. Secretaria de Vigilância em Saúde. v. 48, n. 30, 2017. p. 1-4. Disponível em: <http://portalarquivos2.saude.gov.br/images/pdf/2017/ setembro/21/2017-025-Perfil-epidemiologico-das-tentativas-e-obitos-por-suicidio-no-Brasil-e-a-rede-de-atencao-a-saude.pdf>.

18. Veloso, C. et al. Violência auto infligida por intoxicação exógena em um serviço de urgência e emergência. Revista Gaúcha de Enfermagem 2017; 1-8 (2):38 DOI: http://dx.doi.org/10.1590/1983-1447.2017.02.66187. 19. Oliveira, EM; Félix, TA; Mendonça, CBL; Souza, DR; Ferreira, GB; Freire, MA; Lima, PSF; Teodósio, TBT; Linhares, JM; Almeida, PC. Tentativa de suicídio por intoxicação exógena: contexto de notificações compulsórias. Revista Eletrônica Gestão \& Saúde 2015; 6 (3); 2497-11. Disponível em: <http:// periodicos.unb.br/index.php/rgs/article/view/3125>.

20. Brandenburg, R. et al. In-HospitalMortalityandLong-TermSurvivalofPatientsWithAcutelntoxicationAdmittedtothe ICU. CriticalCare Medicine 2014; 1471-1479. DOl: http://dx.doi.org/10.1097/ccm.0000000000000245. 